\title{
Body Fluid Changes, Cardiovascular Deconditioning and Metabolic Impairment Are Reversed 24 Hours after a 5-Day Dry Immersion
}

\author{
Mickael Coupé ${ }^{1,2}$, Elena Tomilovskaya ${ }^{3}$, Françoise Larcher ${ }^{4}$, Bertrand Diquet ${ }^{5}$, \\ Liudmila Kh. Pastushkova ${ }^{2,3}$, Inesa B. Kozlovskaya ${ }^{3}$, Irina M. Larina ${ }^{2,3}$, Guillemette Gauquelin-Koch ${ }^{6}$, \\ Vladimir A. Kulchitsky ${ }^{7}$, Marc-Antoine Custaud ${ }^{1,2}$, Nastassia M. Navasiolava ${ }^{1,2}$ \\ ${ }^{1}$ CNRS UMR 6214, INSERM U1083, Angers University, Angers, France \\ ${ }^{2}$ Associated French-Russian Laboratory CaDyWEC, Angers, France \& Moscow, Russia \\ ${ }^{3}$ Institute of Biomedical Problems RAS SSC, Moscow, Russia \\ ${ }^{4}$ Biochemistry Laboratory, Angers University Hospital, Angers, France \\ ${ }^{5}$ Pharmacokinetics Laboratory, Angers University Hospital, Angers, France \\ ${ }^{6}$ French Space Agency (CNES), Paris, France \\ ${ }^{7}$ Institute of Physiology, National Academy of Sciences, Minsk, Belarus \\ Email: nastassia.navasiolava@etud.univ-angers.fr
}

Received December 31, 2012; revised February 5, 2013; accepted March 8, 2013

\begin{abstract}
Dry immersion is an effective and useful model for research in physiology and physiopathology. The focus of this study was to provide integrative insight into renal, endocrine, circulatory, autonomic and metabolic effects of dry immersion. We assessed if the principal changes were restored within $24 \mathrm{~h}$ of recovery, and determined which changes were mainly associated with immersion-induced orthostatic intolerance. Five-day dry immersion without countermeasures, and with ad libitum water intake, standardized diet and a permitted short daily rise was performed in a relatively large sample for this experiment type (14 healthy young men). Reduction of total body water derived mostly from extracellular compartment, and stabilized rapidly at the new operating point. Decrease in plasma volume was estimated at $20 \%-25 \%$. Five-day immersion was sufficient to impair metabolism with a decrease in glucose tolerance and hypercholesterolemia, but was not associated with pronounced autonomic changes. Five-day immersion induced marked cardiovascular impairment. Immediately after immersion, over half of the subjects were unable to accomplish the 20 -min $70^{\circ}$ tilt; during tilt, heart rate and total peripheral resistance were increased, and stroke volume was decreased. However, 24 hours of normal physical activity appeared sufficient to reverse orthostatic tolerance and all signs of cardiovascular impairment, and to restitute plasma volume and extracellular fluid volume. Similarly, metabolic impairment was restored. In our study, the major factor responsible for orthostatic intolerance appeared to be hypovolemia. The absence of pronounced autonomic dysfunction might be explained by relatively short duration of dry immersion and daily short-time orthostatic stimulation.
\end{abstract}

Keywords: Physical Inactivity; Modeled Weightlessness; Water-Electrolyte Balance; Body Fluid Compartments; Orthostatic Intolerance

\section{Introduction}

Water immersion has been demonstrated as an efficient and useful model for renal, neuro-hormonal and body fluid physiology [1-3]. Since antiquity, it is known that immersion induces diuresis, indeed the Latin word "urinator" means wisely "diver". However, the water immersion method is generally limited to several hours because of skin maceration. This method has been modified to separate the subject from water using elastic tissue (dry immersion, DI), which allows for longer immersion pe- riods and increased comfort for the subjects [4] (Figure 1). Using longer duration, it becomes possible to study the effects of a new body fluid steady state on major bodily functions.

DI allows for studying of acute expansion of central volume (first hours), maintained hypovolemic state (continuation of DI for days), and acute diminution of central volume (immediate recovery). DI rapidly induces hypovolemia and cardiovascular deconditioning, observed as orthostatic hypotension, increased heart rate and reduced exercise capacity. DI has also been recognized as a 


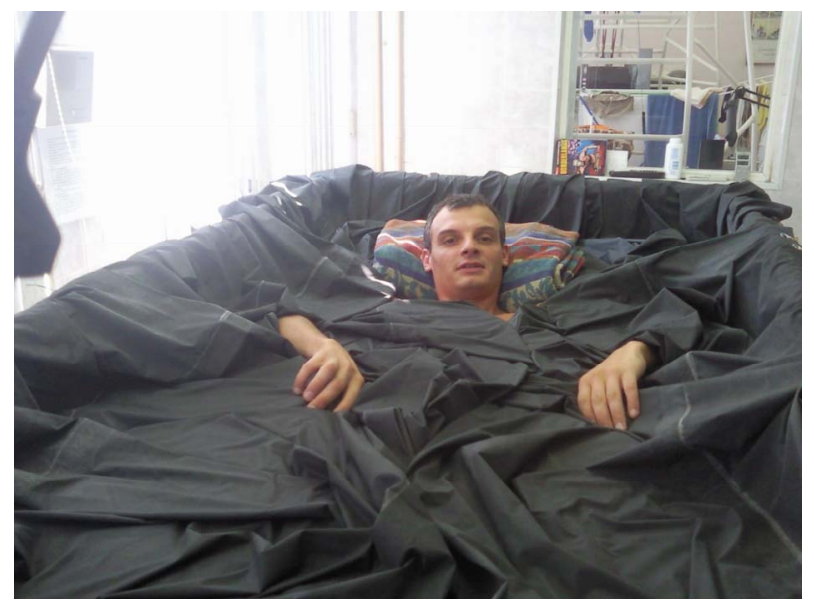

Figure 1. Dry immersion method. The subject is separated from water with waterproof tissue.

valuable model for physical inactivity [5], due to acute limitation of voluntary muscular activity, reduction of gravitational stress on muscles, and deep atonia [4].

The DI model was first developed by Russian physicians and scientists to mimic the space environment. This method, however, might be directly useful in some common disease. DI appeared effective in treatment of refractory edema of different etiologies (cardiovascular diseases, renal pathologies, liver cirrhosis, and burns). In patients with edema, the 1.5 - 3 fold increase in diuresis observed after 4 - 6 hours of DI persisted for 2 days [6, 7]. In patients with treatment-resistant hypertensive crisis, 1.5 hours of DI induced a rapid and prolonged blood pressure reduction and sedation [8]. Together this indicates the interest of DI model for physiology and physiopathology studies.

In DI studies, the volunteers' number rarely exceeds 6 - 8 subjects. We had the unique opportunity to study the effects of DI in a relatively large population $(n=14)$ for this specific protocol. Our first aim was to provide a comprehensive analysis of body fluid, autonomic, cardiovascular and metabolic changes induced by a 5-day DI. We further examined whether the 24-hour recovery period was sufficient to restore the principal changes induced by DI. Finally, we determined the main changes associated with orthostatic intolerance induced by DI.

\section{Methods}

\subsection{Subjects}

The study included a group of 14 healthy non-smoking Russian men (age $22 \pm 1 \mathrm{y}$, height $174 \pm 1 \mathrm{~cm}$, weight $67.9 \pm 1.5 \mathrm{~kg}$; mean $\pm \mathrm{SE}$ ). All volunteers provided written informed consent, in compliance with the Helsinki Declaration. The experimental protocol was approved by the Committee of Biomedicine Ethics of the Russian Academy of Sciences. The subjects were randomly di- vided into 2 groups (group R0, $\mathrm{n}=7$, and group $\mathrm{R}+1, \mathrm{n}=$ 7 ), according to the day of orthostatic test in the recovery period.

\subsection{General Protocol}

The protocol used in this study is schematized in Figure 2. DI was performed for 5 days (120 hours) at the Dry Immersion Facility of the Institute of Biomedical Problems (Moscow, Russia). For baseline and recovery measurements and sampling, ambulatory periods of 3 and 4 days preceded and followed DI, respectively (B-3 to $\mathrm{B}-1$, and $\mathrm{R} 0$ to $\mathrm{R}+3$ ). The subjects were immersed one after another. Thermoneutral water temperature $\left(32^{\circ}\right.$ $33.5^{\circ}$ ) was continuously maintained; air temperature was maintained at $24^{\circ} \mathrm{C}-25^{\circ} \mathrm{C}$, and air humidity was $40 \%$ $50 \%$.

The present study was one of 16 studies conducted during this DI protocol. Some experiments required extraction from bath; in this case, the subjects remained supine on a lifting platform. Total out-of-bath supine time for the $120 \mathrm{~h}$ of immersion was $4.11 \pm 0.15 \mathrm{~h}$ (mean $\pm \mathrm{SE}$ ). In addition, once a day (in the evening) the subjects were allowed to leave the bath for personal hygiene procedures (defecation and shower in a sitting position) and weighing (in a standing position). Total out-of-bath sitting/standing time for the 120 hours of immersion was $1.43 \pm 0.07 \mathrm{~h}($ mean $\pm \mathrm{SE})$. Otherwise, the subjects remained in a supine position during DI for all activities and were continuously observed by video monitoring. Blood pressure and heart rate (using a sphygmomanometer) were measured daily at 8 a.m. The onset and end of the immersion were both at 10 a.m., so morning measurements on day 1 were performed before immersion, and on day R0 - still under immersion. Before, during and after DI, water intake was ad libitum (measured), and diet was standardized. Daily caloric intake was $\sim 2880 \pm 60 \mathrm{kcal}$. Dietary sodium was set to $2.8 \mathrm{mmol} / \mathrm{kg} /$ day, calcium - to $\sim 1100 \mathrm{mg} / \mathrm{day}$, and protein intake to $1.15 \mathrm{~g} / \mathrm{kg} /$ day.

\subsection{Bio-Impedance Measurements}

Bio-impedance measurements by multifrequency impedance device Hydra ECF/ICF 4200 (Xitron Technologies, San Diego, USA) were performed out of the bath in the evening (at 5 p.m.) at baseline (B-2 or B-1), day 1 (7 hours after beginning DI), day 3 , day 5 , R0 ( 7 hours after the end of DI) and R+1. Each measurement was repeated 3 times, and mean values were calculated. We estimated extracellular fluid (ECF), intracellular fluid (ICF) and total body water. Whole body wrist-ankle measurement approach was used. The subject was in supine position for at least 10 minutes prior to the start of measurements, without contact with metal or water. The arms were 


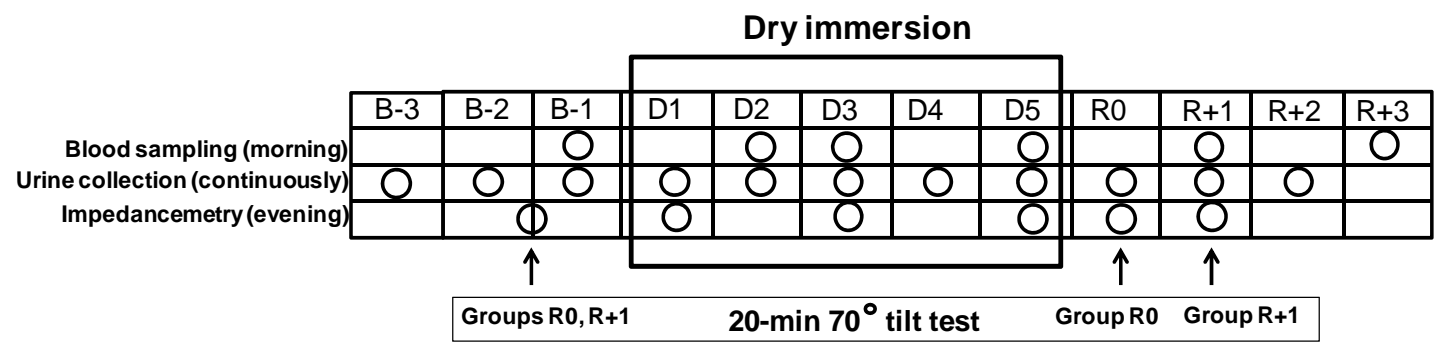

Figure 2. Schematic outlining of the experimental protocol. B-3, B-2 and B-1, baseline days prior to immersion; D1-D5, days of dry immersion; $\mathrm{RO}-\mathrm{R}+3$, recovery days; circles, days of sampling or measurement; arrows, days of tilt-test.

abducted from the body and legs were separated. Four gum-based electrodes measuring $5 \mathrm{~cm}^{2}$ were used: two current injection electrodes on the hand and foot on the dorsal surfaces proximal to the metacarpal-phalangeal and metatarsal-phalangeal joints, respectively, and two voltage detector electrodes on the mid-line between the prominent ends of the radius and ulna of the wrist and mid-line between the medial and lateral malleoli of the ankle.

Both before and after immersion, the subjects were weighed prior to measurements. During immersion, body weight data from the evening of the previous day were used for calculations.

\subsection{Tilt Test}

The head-up tilt test was performed in a quiet room with an ambient air temperature of $24^{\circ} \mathrm{C}-25^{\circ} \mathrm{C}$, in the morning, before immersion (day B-2 or B-1) and immediately after completing the 5-day DI (first rising, group R0) or on the second day of recovery (group $\mathrm{R}+1$ ). The subject was placed on a tilt-table in a supine position, and allowed to rest for at least $30 \mathrm{~min}$; the data were then recorded from this resting supine position for 10 minutes. Afterwards, the subject was tilted $70^{\circ}$ head-up. Subjects were instructed to stand with feet shoulder-width apart, keeping as still as possible to avoid muscle pump activity. Subjects remained in the $70^{\circ}$ head-up tilt (HUT) position with continuous monitoring for $20 \mathrm{~min}$, until presyncope, or until the subject's request to stop.

Standard ECG was recorded continuously, and beat-tobeat R-R intervals and heart rate (HR) were extracted from the signal. Blood pressure was measured continuously on the third finger using a non-invasive finger cuff device (Finapres, Ohmeda, Englewood, Colorado). The finger cuff was maintained at heart level in both supine and standing positions. Additionally, arm blood pressure was measured by Dinamap (Carescape V100, GE Healthcare, Finland) at baseline, and at 5th, 10th and 15th minutes of the HUT. Skin blood flow at the calf level was estimated using Laser-Doppler flowmetry as previously described [9]. Calf skin temperature was controlled by monitoring using a thermocouple connected to an elec- tronic thermometer (BAT-12, Physitemp Instruments, Clifton, New Jersey).

The stable $5 \mathrm{~min}$ of data for each period (basal and end tilt) were averaged and used for analysis. In case of orthostatic intolerance, the 5 minutes prior to intolerance were chosen for analysis [10]. For the single subject who developed orthostatic intolerance at the third minute of tilt, the 2 min mark prior to intolerance was analyzed. Cardiac output (CO), stroke volume (SV), and total peripheral resistance (TPR) were estimated by an analysis of blood pressure waveform.

Analysis of orthostatic responses, power spectrum analysis of heart rate variability, and analysis of the spontaneous baroreflex sensitivity was performed as previously described [10]. Skin vascular resistance (SVR) was calculated by dividing mean blood pressure by skin blood flow. In the tilted position, heart-Laser-Doppler probe distance (hydrostatic component of blood pressure) was taken into account to correct blood pressure at the probe level and calculate SVR.

\subsection{Sample Collection and Analyses}

\subsubsection{Blood Sampling and Estimation of Plasma Volume Dynamics}

Blood sampling was performed with participants in the supine position in the morning before breakfast on baseline (B-1), day 2, day 3, day 5, and the second and fourth recovery days $(\mathrm{R}+1$ and $\mathrm{R}+3)$.

Fasting glucose (using Accu Check Performa glucometer), hemoglobin (using HemoCue hemoglobin analyzer) and hematocrit (using microhematocrit method) were determined immediately after blood collection. Percentage changes in plasma volume were calculated indirectly using:

- $\mathrm{Hb}$ and Hct count (Dill and Costill formula):

$$
\begin{aligned}
\operatorname{DPV}(\%)= & 100 \times[\mathrm{HbB}(1-0.01 \mathrm{Hcti})] / \\
& {[\mathrm{Hbi}(1-0.01 \mathrm{HctB})]-100 ; }
\end{aligned}
$$

- Only Hct count (van Beaumont formula):

$$
\begin{aligned}
\operatorname{DPV}(\%)= & {[100 /(100-\mathrm{HctB})] \times } \\
& {[100 \times(\mathrm{HctB}-\mathrm{Hcti}) / \mathrm{Hcti})] }
\end{aligned}
$$

- Plasma protein count [11]: 


$$
\operatorname{DPV}(\%)=100 \times[\operatorname{Prot} \mathrm{B} / \operatorname{Prot} \mathrm{i}]-100 ;
$$

where HctB, HbB and Prot B are hematocrit, hemoglobin and proteins concentrations at baseline, respectively, and Hcti, Hbi and Prot $\mathrm{i}$ are the corresponding values on day 2 , day 3 , day 5 and $\mathrm{R}+1$.

Plasma and serum samples were analyzed for renin, aldosterone, brain natriuretic peptide (BNP), electrolytes $\left(\mathrm{Na}^{+}, \mathrm{K}^{+}, \mathrm{Cl}^{-}, \mathrm{Ca}^{2+}\right)$, osmolality, proteins, urea and creatinine concentrations, high-sensitivity CRP, insulin, leptin, triglycerides, total cholesterol, and HDL-cholesterol. LDL-cholesterol was calculated using the Friedewald formula. Homeostasis model assessment-insulin resistance index (HOMA-IR) was calculated as fasting insulin concentration $(\mu \mathrm{U} / \mathrm{ml}) \times$ fasting glucose concentration $(\mathrm{mmol} / \mathrm{L}) / 22.5$.

\subsubsection{Urine Sampling}

Urine pools were collected over 24 hours for 3 days prior to immersion (B-3, B-2 and B-1), 5 days of immersion (day 1-day 5 ) and 3 recovery days $(\mathrm{R} 0, \mathrm{R}+1$ and $\mathrm{R}+2)$. Urine volume was measured and the aliquots were stored at $-80^{\circ} \mathrm{C}$. The partial water balance, defined as the difference between consumed water (including drinking water, tea, juice, soup) and urine volume, was calculated. Urine samples were analyzed for electrolytes $\left(\mathrm{Na}^{+}, \mathrm{K}^{+}\right.$, $\mathrm{Cl}^{-}$), osmolality, creatinine, urea and urinary free cortisol (UFC), urinary catecholamines (epinephrine, norepinephrine, metanephrine, normetanephrine, dopamine). Creatinine, free water and osmolal clearances were calculated for days B-2, day 1 , day 2 , day $4, \mathrm{R} 0$ and $\mathrm{R}+2$ using the 24-h urine samples from these days and the morning blood samples from the next days (i.e. B-1, day 2, day 3 , day $5, \mathrm{R}+1, \mathrm{R}+3)$.

\subsubsection{Biochemical Analyses}

Active renin analysis was performed using a chemiluminescence immunoassay on the Liaison analyzer (DiaSorin). BNP was measured using a chemiluminescence immunoassay on the "Architect i2000" analyser (Abbott). Plasma aldosterone was determined by a competition radioimmunoassay using a commercially available RIA kit (Immunotech, Beckman Coulter). Urinary free cortisol was detected by liquid chromatography-tandem mass spectrometry (LC-MS/MS) with an Applied Biosystems/MDS Sciex Api 3000 triple quad mass spectrometer equipped with turbolon spray source. Plasma and urine osmolality were measured by freezing-point depression in comparison to pure water. Urinary catecholamines were determined by high-pressure liquid chromatography.

All the other variables from blood and urine samples were evaluated using the Architect c16000 automated clinical chemistry analyzer (Abbot).

Minimal detectable level for plasma aldosterone and
BNP was $10 \mathrm{ng} / \mathrm{L}$, for serum hs-CRP $-0.1 \mathrm{mg} / \mathrm{L}$, for urinary epinephrine $-2 \mu \mathrm{g} / \mathrm{L}$, and for urinary metanephrine $-0.1 \mu \mathrm{g} / \mathrm{L}$. Results that were less than the minimal detectable level were taken for half minimal value.

\subsection{Data Analysis}

All values are presented as mean $\pm \mathrm{SE}$. The effects of DI were tested with ANOVA for repeated measures. Statistically significant differences were further analyzed by post-hoc pairwise comparisons (Least Significant Difference). Differences were considered to be statistically significant at $\mathrm{p}<0.05$. Analyses were performed using SPSS 15.0 for Windows.

\section{Results}

\subsection{General Data}

Overall, the five-day DI was well tolerated by the study group; all the subjects, however, had a moderate backache at the beginning, which disappeared by the third to fourth day of immersion.

\subsection{Body Fluids and Kidney Regulation}

\subsubsection{Body Fluids}

Plasma volume changes determined by $\mathrm{Hb}$ and Hct count, and by Hct count alone, were similar. Plasma volume decreased by $17 \%$ - $18 \%$ within the first 24 hours of immersion, and remained at reduced level until the end of immersion. Plasma protein count also revealed plasma volume reduction under immersion, but less pronounced, at approximately $7 \%-8 \%$. On the second recovery day, plasma volume as determined by all 3 methods did not differ from baseline (Figure 3). Water intake varied throughout the experiment without significance. Daily diuresis was increased by approximately $90 \%$ on day 1 , and remained elevated throughout the immersion. Partial water balance at day 1 was approximately $1 \mathrm{~L}$ less than at baseline, and remained negative throughout the immersion. On the first recovery day, we observed a compensatory decrease in diuresis and increase in water balance (Table 1). Free water clearance did not significantly change throughout the protocol. Osmolal clearance was decreased during immersion (significantly on day $4 ; p=0.09$ on day 1 and day 2) (Table S1). The dynamics of fluid compartments are shown in Figure 4. Extracellular fluid decreased with the onset of immersion (by $5.1 \% \pm 1.3 \%$ ), with the maximal decrease of $7.8 \% \pm$ $1.4 \%$ on day 3 . In absolute values, the extracellular volume lost on day 3 was approximately $1.4 \pm 0.3 \mathrm{~L}$. Total body water also decreased to approximately $1.6 \mathrm{~L}$ on day 3 , and remained decreased on day 5 . In recovery, and already within 7 hours after the end of DI, the extracellular fluid and total body water did not significantly 


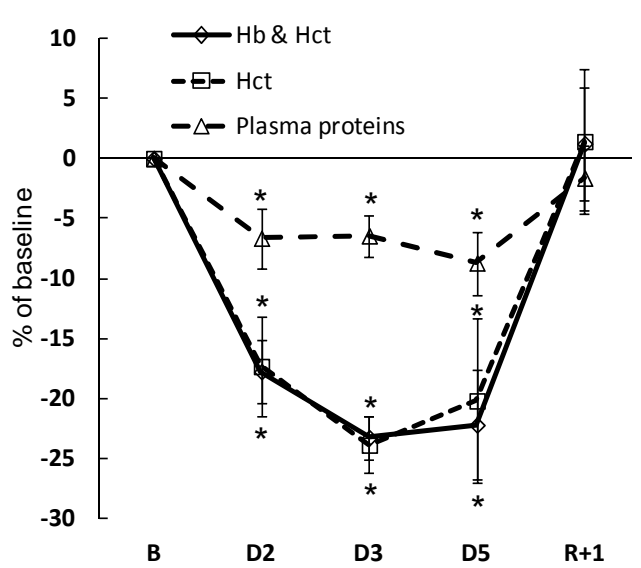

Figure 3. Variations in plasma volume in percentage of baseline, calculated indirectly by hemoglobin and hematocrit count (Hb and Het), hematocrit count alone (Hct), and plasma proteins count. B: baseline; D: dry immersion; $\mathrm{R}$ : recovery. Values are means $\pm \mathrm{SE}$; $" \mathrm{p}<0.05$ vs baseline.

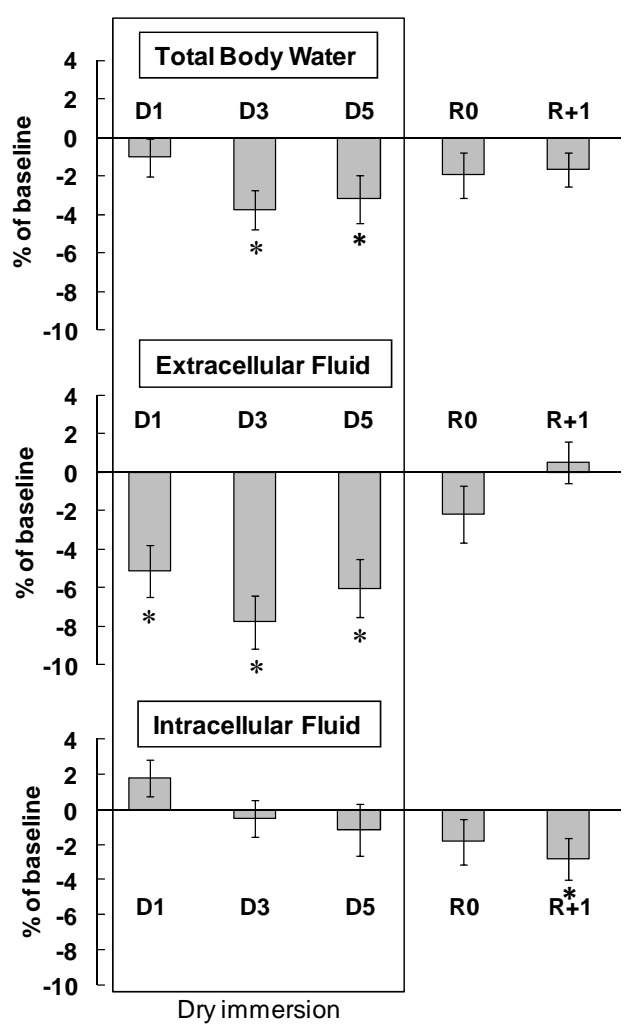

Figure 4. Variations of fluid compartments. D: dry immersion; R: recovery. Values are means $\pm \mathrm{SE}$; $\mathrm{p}<0.05$ vs baseline.

differ from baseline levels. Intracellular fluid showed non-significant variations during DI and was decreased by approximately $3 \%$ at $\mathrm{R}+1$.

\subsubsection{Blood and Urine Biochemistry}

Blood levels of $\mathrm{Na}^{+}, \mathrm{K}^{+}, \mathrm{Cl}^{-}, \mathrm{Ca}^{2+}$, proteins, urea, creatinine and osmolality remained within normal values at all measurements. DI was accompanied by a significant increase in blood $\mathrm{K}^{+}, \mathrm{Ca}^{2+}$ and protein content. Blood urea was slightly decreased at the beginning of DI, and slightly increased 24 hours after the end of DI ( Table 2).

Urinary sodium loss increased at the beginning of DI (with a two-fold increase on day 1), followed by decrease in recovery (with a 3-fold decrease on $\mathrm{R} 0$ ). The dynamics of urinary $\mathrm{Cl}^{-}$excretion showed similar trends. Urinary potassium excretion was not significantly changed. The urinary $\mathrm{Na}^{+} / \mathrm{K}^{+}$ratio increased significantly only on day 1 and did not differ from the baseline during the rest of immersion. The end of immersion was accompanied by a decrease in the $\mathrm{Na}^{+} / \mathrm{K}^{+}$ratio (Table 1).

Urinary osmolality was reduced during immersion, with a significant reduction on day 3 , and tendency to reduction - with $\mathrm{p}=0.07$ to 0.09 - on days 1,2 and 4 , with no significant difference compared to baseline in recovery (Table 1). Urinary urea and urinary creatinine did not change significantly (Table 1). Urea clearance increased on day 1 and decreased on R0. Creatinine clearance did not change throughout the DI (Table S1).

\subsection{Cardiovascular System at Rest}

\subsubsection{Morning Heart Rate and Blood Pressure}

Heart rate and blood pressure remained within normal limits. Heart rate was slightly reduced at the beginning of DI, and systolic blood pressure was slightly lower at the end of DI (Table S2).

\subsubsection{Cardiovascular Hormones Regulating Volemia}

Twenty-four hours after the onset of DI, plasma renin decreased two-fold and plasma BNP increased fourfold. Plasma aldosterone was not significantly changed. Twenty-four hours after the end of DI, we observed a two-fold increase in renin and 3-fold increase in aldosterone. On the fourth recovery day, renin and aldosterone were not modified, and BNP was significantly increased (Figure 5).

\subsection{Cardiovascular Variables during Tilt Test}

\subsubsection{Tolerance}

Before DI, only one subject (assigned to group R0) out of 14 was unable to complete the 20-min HUT. After DI, on day R0, 4 out of 7 subjects were non-finishers. On day $\mathrm{R}+1$, all subjects finished the test.

\subsubsection{Hemodynamic Responses to Tilt Test: Supine and Upright Position}

The hemodynamic responses to the tilt test are presented in Table 3. Measurements on R0 compared to preimmersion, performed in the supine position, showed a 
Table 1. Urine biochemistry, diuresis, water intake and partial water balance before, during and following dry immersion.

\begin{tabular}{|c|c|c|c|c|c|c|c|c|c|c|c|}
\hline & B-3 & B-2 & B-1 & DI 1 & DI 2 & DI 3 & DI 4 & DI 5 & R0 & $\mathrm{R}+1$ & $\mathrm{R}+2$ \\
\hline $\mathrm{K}^{+}, \mathrm{mmol} / 24 \mathrm{~h}$ & $35 \pm 6$ & $35 \pm 5$ & $39 \pm 6$ & $40 \pm 2$ & $41 \pm 4$ & $45 \pm 6$ & $47 \pm 5$ & $55 \pm 6$ & $38 \pm 4$ & $42 \pm 3$ & $51 \pm 4$ \\
\hline $\mathrm{Na}^{+}, \mathrm{mmol} / 24 \mathrm{~h}$ & $132 \pm 12$ & $120 \pm 15$ & $123 \pm 21$ & $211 \pm 17^{*}$ & $163 \pm 17^{*}$ & $131 \pm 13$ & $137 \pm 15$ & $157 \pm 14$ & $46 \pm 4^{*}$ & $70 \pm 8$ & $113 \pm 19$ \\
\hline $\mathrm{Na}^{+} / \mathrm{K}^{+}$ratio & $3.8 \pm 0.4$ & $3.8 \pm 0.4$ & $3.4 \pm 0.6$ & $5.6 \pm 0.7^{*}$ & $4.0 \pm 0.3$ & $3.2 \pm 0.3$ & $3.0 \pm 0.2$ & $3.0 \pm 0.2$ & $1.5 \pm 0.2^{*}$ & $1.8 \pm 0.3^{*}$ & $2.1 \pm 0.3$ \\
\hline $\mathrm{Cl}^{-}, \mathrm{mmol} / 24 \mathrm{~h}$ & $132 \pm 13$ & $108 \pm 13$ & $121 \pm 21$ & $181 \pm 13^{*}$ & $160 \pm 18^{*}$ & $128 \pm 12$ & $130 \pm 15$ & $147 \pm 14$ & $54 \pm 4^{*}$ & $61 \pm 7^{*}$ & $111 \pm 17$ \\
\hline $\begin{array}{l}\text { Creatinine, } \\
\mathrm{mmol} / 24 \mathrm{~h}\end{array}$ & $9.1 \pm 1.2$ & $8.7 \pm 1.0$ & $8.9 \pm 1.4$ & $9.1 \pm 0.6$ & $9.3 \pm 1.0$ & $9.5 \pm 1.2$ & $8.5 \pm 0.8$ & $10.8 \pm 1.2$ & $7.4 \pm 0.7$ & $7.8 \pm 0.6$ & $8.2 \pm 0.9$ \\
\hline Urea, $\mathrm{mmol} / 24 \mathrm{~h}$ & $209 \pm 25$ & $208 \pm 29$ & $203 \pm 30$ & $231 \pm 20$ & $205 \pm 23$ & $231 \pm 24$ & $223 \pm 18$ & $275 \pm 21$ & $171 \pm 20$ & $210 \pm 13$ & $252 \pm 30$ \\
\hline $\begin{array}{c}\text { Osmolality, } \\
\mathrm{mOS} / \mathrm{kg}\end{array}$ & $496 \pm 56$ & $497 \pm 42$ & $487 \pm 56$ & $360 \pm 27$ & $375 \pm 22$ & $348 \pm 40^{*}$ & $377 \pm 40$ & $386 \pm 52$ & $534 \pm 43$ & $502 \pm 59$ & $537 \pm 29$ \\
\hline UFC $(\mu \mathrm{g} / 24 \mathrm{~h})$ & $33 \pm 7$ & $20 \pm 4$ & $28 \pm 4$ & $46 \pm 13$ & $46 \pm 8^{*}$ & $40 \pm 5$ & $44 \pm 8^{*}$ & $35 \pm 6$ & $22 \pm 4$ & $20 \pm 2$ & $36 \pm 6$ \\
\hline
\end{tabular}

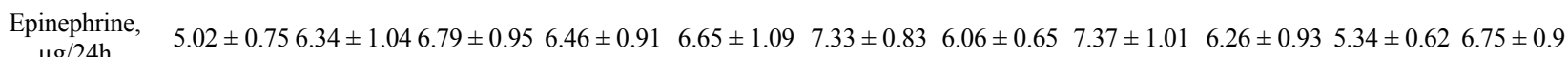
$\mu \mathrm{g} / 24 \mathrm{~h}$

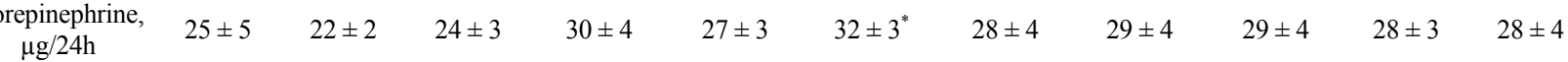

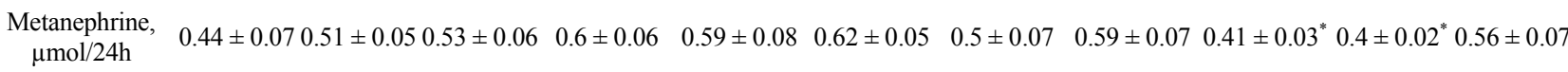

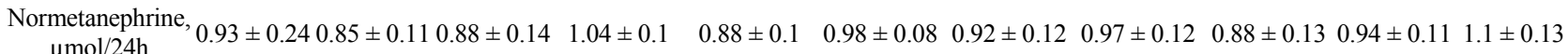

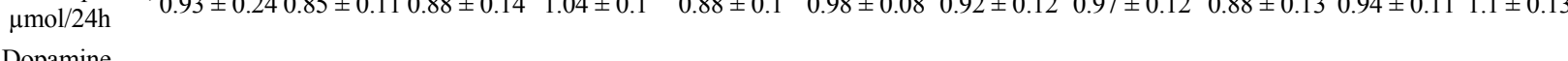

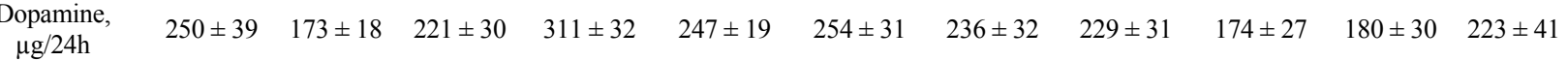

Diuresis, $1 / 24 h \quad 1.37 \pm 0.221 .13 \pm 0.1 \quad 1.23 \pm 0.152 .31 \pm 0.21^{*} 1.94 \pm 0.19^{*} 2.24 \pm 0.34^{*} 1.99 \pm 0.2^{*} 2.26 \pm 0.31^{*} 0.91 \pm 0.11^{*} 1.06 \pm 0.1 \quad 1.23 \pm 0.13$

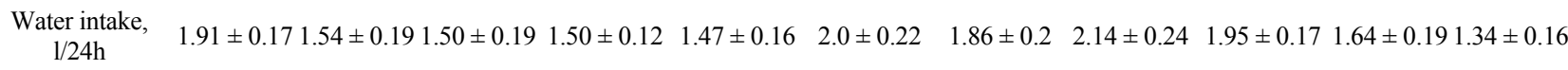

$\begin{aligned} & \text { Partial water } \\ & \text { balance, } \mathrm{ml} / 24 \mathrm{~h}\end{aligned} 770 \pm 210 \quad 420 \pm 190 \quad 320 \pm 110-780 \pm 170^{*}-520 \pm 110^{*}-230 \pm 180^{*}-210 \pm 150^{*}-230 \pm 160^{*} 1030 \pm 180^{*} 590 \pm 160 \quad 200 \pm 85$

B: baseline; DI: dry immersion; R: recovery; UFC: urinary free cortisol; values are means \pm SE, ${ }^{*} \mathrm{p}<0.05$ vs $\mathrm{B}-1$.

Table 2. Blood biochemistry before, during and following dry immersion.

\begin{tabular}{|c|c|c|c|c|c|c|}
\hline & B-1 & DI 2 & DI 3 & DI 5 & $\mathrm{R}+1$ & $\mathrm{R}+3$ \\
\hline $\mathrm{Na}^{+}, \mathrm{mmol} / \mathrm{l}$ & $136 \pm 2$ & $138 \pm 2$ & $139 \pm 3$ & $137 \pm 2$ & $135 \pm 2$ & $136 \pm 2$ \\
\hline $\mathrm{K}^{+}, \mathrm{mmol} / 1$ & $3.6 \pm 0.1$ & $4.0 \pm 0.1^{*}$ & $4.1 \pm 0.1^{*}$ & $4.0 \pm 0.1^{*}$ & $3.7 \pm 0.1$ & $3.7 \pm 0.1$ \\
\hline $\mathrm{Cl}^{-}, \mathrm{mmol} / 1$ & $103 \pm 2$ & $104 \pm 1$ & $105 \pm 2$ & $102 \pm 1$ & $102 \pm 1$ & $103 \pm 2$ \\
\hline $\mathrm{Ca}^{2+}, \mathrm{mmol} / 1$ & $2.14 \pm 0.05$ & $2.22 \pm 0.04^{*}$ & $2.23 \pm 0.05^{*}$ & $2.27 \pm 0.05^{*}$ & $2.18 \pm 0.04$ & $2.08 \pm 0.05$ \\
\hline Proteins, g/1 & $65 \pm 2$ & $70 \pm 2^{*}$ & $70 \pm 2^{*}$ & $72 \pm 2^{*}$ & $67 \pm 2$ & $64 \pm 2$ \\
\hline Urea, mmol/1 & $4.5 \pm 0.3$ & $3.6 \pm 0.2^{*}$ & $4.1 \pm 0.2$ & $4.5 \pm 0.3$ & $5.4 \pm 0.3^{*}$ & $5.2 \pm 0.4$ \\
\hline Creatinine, $\mu \mathrm{mol} / 1$ & $74 \pm 2$ & $72 \pm 2$ & $75 \pm 3$ & $76 \pm 2$ & $77 \pm 3$ & $72 \pm 2$ \\
\hline Osmolality, mOS/kg & $288 \pm 7$ & $283 \pm 3$ & $283 \pm 4$ & $290 \pm 4$ & $284 \pm 3$ & $284 \pm 6$ \\
\hline hs-CRP, mg/l & $0.35 \pm 0.08$ & $0.43 \pm 0.12$ & $0.52 \pm 0.14$ & $0.4 \pm 0.09$ & $0.47 \pm 0.09$ & $0.72 \pm 0.22$ \\
\hline Fasting glucose, $\mathrm{mmol} / 1$ & $4.97 \pm 0.11$ & $4.96 \pm 0.08$ & $4.99 \pm 0.08$ & $4.82 \pm 0.08$ & $4.86 \pm 0.11$ & $4.66 \pm 0.09$ \\
\hline HOMA-IR & $1.27 \pm 0.13$ & $1.59 \pm 0.11^{*}$ & $1.59 \pm 0.09$ & $1.76 \pm 0.15^{*}$ & $1.35 \pm 0.16$ & $1.11 \pm 0.17$ \\
\hline Leptin, ng/ml & $2.23 \pm 0.31$ & $2.49 \pm 0.28^{*}$ & $2.73 \pm 0.38^{*}$ & $2.66 \pm 0.33$ & $2.05 \pm 0.22$ & $2.75 \pm 0.55$ \\
\hline Total cholesterol, mmol/1 & $3.71 \pm 0.18$ & $4.14 \pm 0.18^{*}$ & $4.31 \pm 0.23^{*}$ & $4.52 \pm 0.31^{*}$ & $3.61 \pm 0.2$ & $3.53 \pm 0.17$ \\
\hline HDL cholesterol, mmol/1 & $1.23 \pm 0.08$ & $1.32 \pm 0.08^{*}$ & $1.28 \pm 0.07$ & $1.32 \pm 0.1^{*}$ & $1.12 \pm 0.08$ & $1.15 \pm 0.07$ \\
\hline LDL cholesterol, mmol/1 & $2.11 \pm 0.17$ & $2.41 \pm 0.16^{*}$ & $2.59 \pm 0.21^{*}$ & $2.76 \pm 0.27^{*}$ & $2.04 \pm 0.16$ & $2.03 \pm 0.14$ \\
\hline Triglycerides, mmol/1 & $0.81 \pm 0.11$ & $0.86 \pm 0.12$ & $0.96 \pm 0.06$ & $0.97 \pm 0.07$ & $0.97 \pm 0.09$ & $0.76 \pm 0.07$ \\
\hline
\end{tabular}

B: baseline; DI: dry immersion; R: recovery; values are means \pm SE, ${ }^{*} \mathrm{p}<0.05$ vs baseline. 
Table 3. Hemodynamic and autonomic responses to tilt test.

\begin{tabular}{|c|c|c|c|c|c|c|c|c|c|c|}
\hline \multicolumn{6}{|c|}{ Group R0, $\mathrm{n}=7$} & \multicolumn{5}{|c|}{ Group $\mathrm{R}+1, \mathrm{n}=7$} \\
\hline & $\mathrm{B}$ & $\mathrm{n}$ & R0 & $\mathrm{n}$ & $\begin{array}{l}\text { P value, } \\
B \text { vs R0 }\end{array}$ & B & $\mathrm{n}$ & $\mathrm{R}+1$ & $\mathrm{n}$ & $\begin{array}{l}\mathrm{p} \text { value, } \\
\mathrm{B} \text { vs } \mathrm{R}+1\end{array}$ \\
\hline \multicolumn{11}{|c|}{ Supine } \\
\hline $\mathrm{HR}, \mathrm{bpm}$ & $70.3 \pm 6.5$ & 7 & $83.1 \pm 6.4^{*}$ & 7 & 0.017 & $61.9 \pm 1.6$ & 7 & $60.1 \pm 2.8^{\ddagger}$ & 7 & 0.457 \\
\hline SBP, mmHg & $112.7 \pm 2.5$ & 7 & $124.0 \pm 4.2^{*}$ & 7 & 0.034 & $113.6 \pm 4.2$ & 7 & $114.7 \pm 4.9$ & 7 & 0.750 \\
\hline $\mathrm{DBP}, \mathrm{mmHg}$ & $56.7 \pm 3.6$ & 7 & $69.3 \pm 4.1$ & 7 & 0.058 & $57.0 \pm 4.3$ & 7 & $55.7 \pm 3.2^{\ddagger}$ & 7 & 0.555 \\
\hline $\mathrm{SV}, \mathrm{ml}$ & $86.9 \pm 5.9$ & 7 & $64.7 \pm 2.3^{*}$ & 7 & 0.01 & $86.0 \pm 3.3$ & 7 & $93.3 \pm 5.0^{\ddagger}$ & 7 & 0.102 \\
\hline $\mathrm{CO}, 1 / \mathrm{min}$ & $6.04 \pm 0.64$ & 7 & $5.28 \pm 0.33$ & 7 & 0.148 & $5.31 \pm 0.26$ & 7 & $5.63 \pm 0.45$ & 7 & 0.233 \\
\hline TPR, AU & $0.876 \pm 0.077$ & 7 & $1.173 \pm 0.138$ & 7 & 0.061 & $0.894 \pm 0.057$ & 7 & $0.909 \pm 0.091$ & 7 & 0.871 \\
\hline SVR, AU & $8.6 \pm 1.1$ & 7 & $19.8 \pm 4.3$ & 7 & 0.063 & $11.7 \pm 1.9$ & 7 & $10.2 \pm 2.0$ & 7 & 0.555 \\
\hline LF RRi normalized & $0.63 \pm 0.08$ & 7 & $0.75 \pm 0.03$ & 5 & 0.115 & $0.62 \pm 0.04$ & 7 & $0.60 \pm 0.06$ & 7 & 0.852 \\
\hline LF/HF RRi & $2.65 \pm 0.81$ & 7 & $3.28 \pm 0.43$ & 5 & 0.983 & $1.80 \pm 0.29$ & 7 & $1.97 \pm 0.60$ & 7 & 0.786 \\
\hline SBRs, $\mathrm{ms} / \mathrm{mmHg}$ & $17.1 \pm 2.2$ & 7 & $13.4 \pm 2.4$ & 7 & 0.207 & $18.2 \pm 2.2$ & 6 & $16.8 \pm 1.7$ & 6 & 0.496 \\
\hline \multicolumn{11}{|c|}{ End tilt } \\
\hline $\mathrm{HR}, \mathrm{bpm}$ & $97.9 \pm 7.1^{\dagger}$ & 7 & $127.4 \pm 6.2^{* \dagger}$ & 7 & 0.001 & $92.6 \pm 4.3^{\dagger}$ & 7 & $95.5 \pm 3.7^{\ddagger \dagger}$ & 7 & 0.477 \\
\hline SBP, mmHg & $114.4 \pm 2.5$ & 7 & $116.4 \pm 3.2$ & 7 & 0.586 & $118.9 \pm 3.9$ & 7 & $119.0 \pm 4.9$ & 7 & 0.975 \\
\hline DBP, mmHg & $60.0 \pm 2.9$ & 7 & $70.6 \pm 2.1^{*}$ & 7 & 0.019 & $70.7 \pm 4.3^{\dagger}$ & 7 & $70.9 \pm 3.6^{\dagger}$ & 7 & 0.970 \\
\hline $\mathrm{SV}, \mathrm{ml}$ & $45.8 \pm 4.0^{\dagger}$ & 7 & $31.8 \pm 1.7^{* \dagger}$ & 6 & 0.024 & $54.4 \pm 5.8^{\dagger}$ & 7 & $54.5 \pm 2.3^{+\dagger}$ & 7 & 0.988 \\
\hline $\mathrm{CO}, 1 / \mathrm{min}$ & $4.30 \pm 0.13^{\dagger}$ & 7 & $4.12 \pm 0.30$ & 6 & 0.867 & $4.98 \pm 0.49$ & 7 & $5.18 \pm 0.29^{\ddagger}$ & 7 & 0.693 \\
\hline TPR, AU & $1.498 \pm 0.099^{\dagger}$ & 7 & $1.692 \pm 0.176^{\dagger}$ & 6 & 0.469 & $1.087 \pm 0.083$ & 6 & $1.139 \pm 0.051^{\text {t† }}$ & 6 & 0.603 \\
\hline SVR, AU & $36.1 \pm 5.8^{\dagger}$ & 7 & $37.2 \pm 5.5$ & 6 & 0.567 & $45.5 \pm 7.9^{\dagger}$ & 6 & $40.3 \pm 11.7^{\dagger}$ & 7 & 0.966 \\
\hline LF RRi normalized & $0.80 \pm 0.06$ & 7 & $0.81 \pm 0.04^{\dagger}$ & 4 & 0.182 & $0.80 \pm 0.03^{\dagger}$ & 7 & $0.82 \pm 0.03^{\dagger}$ & 7 & 0.562 \\
\hline LF/HF RRi & $6.63 \pm 1.66^{\dagger}$ & 7 & $4.97 \pm 0.76^{\dagger}$ & 4 & 0.099 & $4.61 \pm 0.70^{\dagger}$ & 7 & $5.75 \pm 1.01^{\dagger}$ & 7 & 0.394 \\
\hline SBRs, $\mathrm{ms} / \mathrm{mmHg}$ & $6.0 \pm 0.8^{\dagger}$ & 7 & $4.3 \pm 0.5^{\dagger}$ & 6 & 0.072 & $6.1 \pm 0.9^{\dagger}$ & 7 & $5.2 \pm 0.4^{\dagger}$ & 7 & 0.227 \\
\hline
\end{tabular}

B: baseline; R: recovery; n: subjects number; HR: heart rate; SBP: systolic blood pressure; DBP: diastolic blood pressure; SV: stroke volume; CO: cardiac output; TPR: total peripheral resistance; SVR: skin vascular resistance; LF: low frequency band; HF: high frequency band; RRi: R-R interval; LF/HF: sympathetic index; SBRs: spontaneous baroreflex sensitivity; values are mean $\pm \mathrm{SE} ;{ }^{*} \mathrm{p} \leq 0.05$ vs before; ${ }^{\dagger} \mathrm{p} \leq 0.05$ vs supine; ${ }^{\ddagger} \mathrm{p} \leq 0.05$ vs group.

significant increase in HR and SBP, and a tendency towards increase for DBP $(p=0.058)$, TPR $(p=0.06)$ and SVR (more than 2-fold increase, $p=0.063$ ). SV was decreased, and CO remained unchanged. Measurements taken in the upright position showed increased HR and DBP and decreased SV after immersion, whereas SBP, TPR, SVR and CO remained unchanged.

On $\mathrm{R}+1$, all measured hemodynamic variables did not show significant difference from pre-immersion levels, in both supine and upright positions.

SVR in the upright position was significantly higher than in the supine position for all measurements except those taken on R0.

\subsection{Autonomic Nervous System}

\subsubsection{Catecholamines and Their Metabolites}

Urinary epinephrine and normetanephrine were not significantly changed throughout the experiment. Urinary metanephrine decreased during recovery. Urinary norepinephrine showed a tendency towards increase during the first half of immersion (day $1, \mathrm{p}=0.076$; day $2, \mathrm{p}=$ 0.072 ; day $3, \mathrm{p}<0.05$ ). Urinary dopamine also tended to increase during the first half of immersion (day $1, \mathrm{p}=$ 


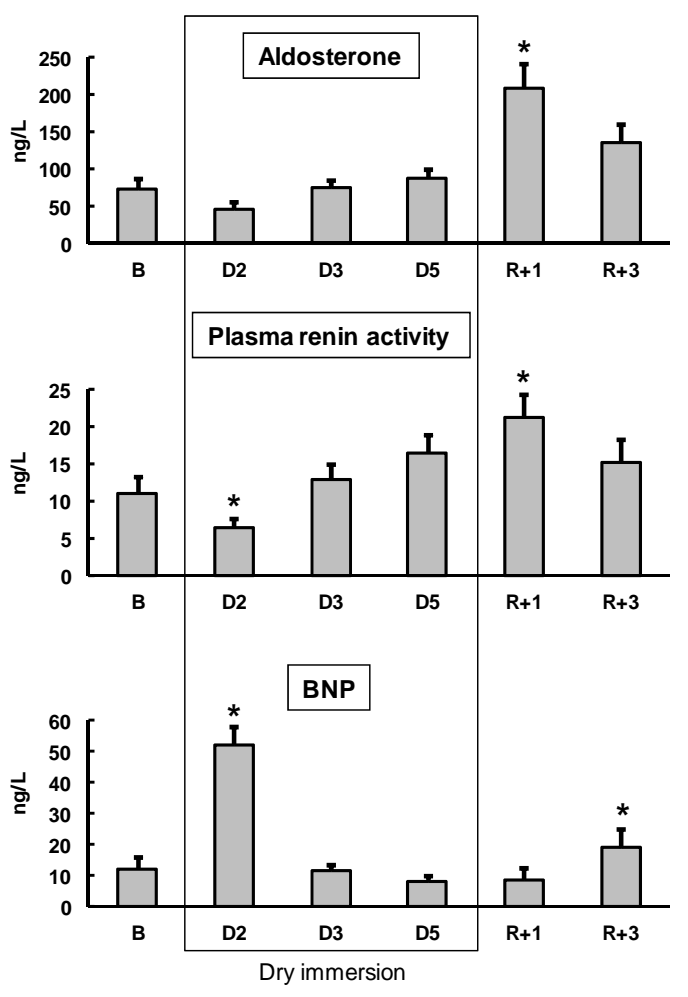

Figure 5. Blood aldosterone, renin and brain natriuretic peptide (BNP) before, during and following dry immersion. B: baseline; D: dry immersion; R: recovery. Values are means $\pm \mathrm{SE} ;{ }^{*} \mathrm{p}<0.05$ vs baseline.

0.08 ; day $2, \mathrm{p}=0.09)$, with tendency to decrease on $\mathrm{R} 0$ $(\mathrm{p}=0.07)($ Table 1).

\subsubsection{Cardiac Autonomic Neural Control}

There was no significant difference in heart rate variability spectrum (LF, LF/HF) before and after DI (both R0 and $\mathrm{R}+1$ ) (Table 3). Spontaneous baroreflex slope in upright position tended towards decrease on R0, but not on $\mathrm{R}+1$ (Table 3).

\subsection{Metabolism}

\subsubsection{Weight Changes}

Body weight during immersion decreased by approximately $700-800 \mathrm{~g}$. This decrease was significant on day 2 and day 3 , with day 4 and day 5 showing a tendency towards decrease $(p=0.06)$. During recovery, there was no significant variation in body weight.

\subsubsection{Blood Variables Relevant to Metabolism}

All studied blood variables relevant to metabolism were within normal limits. Insulin, estimated insulin resistance (HOMA-IR), leptin and both LDL- and HDL-cholesterol fractions increased during DI. On the second recovery day, these variables did not differ from baseline. Fasting glucose and triglyceride levels were not significantly altered during DI (Table 2).

\subsubsection{UFC and hs-CRP}

Urinary free cortisol was slightly increased during immersion (significantly on day 2 and day 4), and unmodified in recovery (Table 1).

hs-CRP was unmodified (Table 2).

\section{Discussion}

Our results showed that during a 5-day DI, the decrease in total body water $(3 \%-4 \%)$ comes mostly from extracellular compartment (decrease 6\% - 8\%), and shows rapid stabilization at the new level. Five days of DI are sufficient to impair metabolism with decrease in glucose tolerance and hypercholesterolemia, but are not associated with pronounced autonomic changes. The 5-day DI induces marked cardiovascular impairment with altered orthostatic tolerance at first rising, which is quickly reversed (within 24 hours upon resuming normal activities).

\subsection{Regulation of Body Fluids: Excretion and Redistribution}

Extracellular osmolality, approximated by blood osmolality, remained stable under DI, suggesting no important changes regarding the intracellular volume. The main changes detected in response to the DI involved extracellular fluid, namely intravascular and interstitial volume.

\subsubsection{Intravascular Volume: Water Loss by Neuroendocrine Regulation}

Fundamental studies of body fluid regulation during short-term water immersion were performed by GauerHenry and Epstein [2]. Water-electrolyte changes in immersion are triggered by congestion of the central vascular regions: hydrostatic compression lowers the peripheral vascular capacity and shifts Starling balance towards increased absorption of interstitial fluid (hemodilution), leading to central hypervolemia. Twenty-four hours after the onset of DI, hormonal regulation was still in process (confirmed by a 2-fold renin decrease and 4-fold BNP increase), but after 48 hours of immersion, the major redistributions were over and hormonal concentrations remained at pre-immersion levels until the end of DI. The increase in BNP on the fourth recovery day may be not directly related to body fluid regulation, but rather reflect increased heart load after a period of enhanced physical inactivity during DI. Previously, we observed a similar increase in NT-proBNP, a proxy measure of BNP, on the fourth recovery day after 7-day DI [12]. The absence of BNP increase in first 24 hours of recovery may reflect the necessity to retain water and compensate for 
hypovolemia, which is prioritized over the need to "unload" the deconditioned heart.

\subsubsection{Interstitial Volume: Water Redistribution by Protein Transfer}

Plasma volume reduction calculated using plasma proteins consisted of as little as $1 / 3$ of that calculated using $\mathrm{Hct}$ or $\mathrm{Hb}$ and Hct. This difference might be explained by the partial transfer of albumins (which make up approximately $60 \%$ of total protein pool) to interstitial fluid. During immersion, with compression of superficial tissues and continuous transcapillary "autotransfusion" [13], enhanced loss of interstitial fluid occurs. To limit tissue dehydration, plasma albumins might shift to interstitial fluid and increase its oncotic pressure. The partial transfer of proteins from plasma to interstitial fluid prevents the further decrease in interstitial fluid volume, which ensures the limitation of liquid loss and its adequate redistribution between intra-and extravascular departments [14]. Plasma proteins can be deposited in extravascular fluid compartments, and, if necessary, quickly return to restore the plasma protein loss. This was demonstrated by experiments with dry immersion and head-down bed rest, in which the plasma protein pool was restored during recovery at a rate exceeding the possibility of its synthesis de novo [15].

\subsection{Hemodynamics}

Five days of DI induced marked impairment of the cardiovascular system with tachycardia and stroke volume diminution both during resting and orthostatic challenge. The increase in HR, TPR and skin vasoconstriction, observed during first rising, might help to maintain blood pressure in conditions of decreased blood volume. The over two-fold increase in supine SVR together with the lack of further increase in SVR when upright immediately after DI might reflect the tension of systems maintaining cerebral blood perfusion.

\subsection{Autonomic Nervous System}

Dry immersion conditions might, on one hand, increase sympathetic activity due to hypovolemia and stress, and on the other hand, decrease it due to physical inactivity. Most DI studies suggest that DI conditions increase sympathetic activation [4]. However, we did not observe pronounced autonomic changes. Heart rate variability remained unmodified, though baroreflex sensitivity was somewhat weakened. The concentrations of urinary cathecolamines and theirs metabolites were not seriously altered.

\subsection{Metabolism}

The metabolic effects of DI are mainly related to the en- forced physical inactivity. DI rapidly impaired glucose metabolism and lipid profile, inducing a decrease in insulin sensitivity and dyslipidemia. The same changes were observed in bed rest experiments [16-18]. It is important to note that physical inactivity for even a short-time is sufficient to impair metabolism ([18], this study). The increase in UFC during DI may be related to increased diuresis, as UFC and cortisone excretion depends on urine volume [19]. It also might reflect stress induced by new environment and the efforts of the organism to preserve homeostasis. Unmodified hs-CRP suggests the absence of a pronounced inflammatory state in DI.

\subsection{Hypovolemia as Major Reason for DI-Induced Cardiovascular Deconditioning}

A number of mechanisms may contribute to cardiovascular deconditioning induced by DI: circulatory blood volume loss [20,21], impaired baroreflex sensitivity [22, 23], autonomic dysfunction [22,24], increased venous compliance [21] enforced by decreased muscle tone [25], macrovascular [26] and microvascular [27] impairment promoted by physical inactivity and prolonged decrease of shear stress forces [28], myocardial impairment, hormonal changes, vestibular changes, or metabolic changes. Hypovolemia appears to be the major contributor for the observed rapid cardiovascular impairment in DI. The importance of hypovolemia is shown by inverse relationships between microgravity-induced plasma volume reduction and orthostatic tolerance [29]. Coyle et al. [30] showed that the decline in $\mathrm{SV}$ and $\stackrel{\circ}{\mathrm{V}} \mathrm{O}_{2}$ max and the increase in HR and TPR during upright exercise following a few weeks of inactivity (only sedentary daily routines and no training) in men who had been training intensely for several years, was largely due to a decline in blood volume. When blood volume was expanded by dextran solution, the reduction of cardiovascular function after a few weeks of detraining was minimal. It is interesting to compare our study, in which plasma volume was normalized spontaneously, with studies in which plasma volume was restored after a prescription. Plasma volume normalization with salt tablets and water after a 12-day head down bed rest (HDBR) prevented orthostatic hypotension and changes in supine hemodynamics and endocrine variables, but was not effective to prevent tachycardia and epinephrine hyperresponsiveness to upright tilt [31]. Plasma volume restoration with fludrocortisone (a synthetic mineralocorticoid) after a 7-day HDBR significantly limited orthostatic intolerance (only one out of 7 subjects remained intolerant), and prevented changes in the baroreflex sensitivity [32]. However, while use of thigh cuffs during a 7-day HDBR significantly (but not completely) limited the decrease in 
plasma volume and the baroreflex sensitivity, it was unable to prevent orthostatic intolerance [33].

In summary, our results suggest that dry immersion induces remarkable changes at renal, endocrine, circulatory and metabolic levels, with pronounced orthostatic intolerance related mainly to hypovolemia. These changes are characterized by both a very rapid onset and very rapid restoration within return to normal activities.

\subsection{Perspectives}

DI is very original and efficient tool for physiological and physiopathological studies, especially in renal and body-fluid domain. In healthcare context, pronounced diuretic, sedative and muscle tone-decreasing effects of DI make it therapeutically promising. DI had already been tested in nephrology and cardiology for treatment of hypertension and edemas, in pediatrics for rehabilitation of preterm infants, and in neurology for muscle spasm relief. It may appear an efficient, easy and low-cost method of non-drug treatment. But to consider larger clinical implementation of DI, it is essential to dispose comprehensive detailed information concerning its effects. Our study on healthy volunteers aimed to contribute to this general knowledge. This work should be continued to further elucidate the mechanisms and potential applications of DI.

\section{Acknowledgements}

The authors thank all the volunteers for their enthusiastic participation in this study.

This study was supported by CNES, CNRS, the regional council "Région des Pays de la Loire" (DC-VACE project), RFFI, and the European FEDER program (Televasc program).

\section{REFERENCES}

[1] M. Epstein, "Cardiovascular and Renal Effects of HeadOut Water Immersion in Man: Application of the Model in the Assessment of Volume Homeostasis," Circulation Research, Vol. 39, No. 5, 1976, pp. 619-628. doi:10.1161/01.RES.39.5.619

[2] M. Epstein, "Renal, Endocrine and Hemodynamic Effects of Water Immersion in Humans," In: M. J. Fregly and C. M. Blatteis, Eds., Handbook of Physiology, Environmental Physiology, Oxford University Press, Oxford, 1996, pp. 845-853.

[3] J. E. Greenleaf, "Physiological Responses to Prolonged Bed Rest and Fluid Immersion in Humans," Journal of Applied Physiology, Vol. 57, No. 3, 1984, pp. 619-633.

[4] N. M. Navasiolava, M. A. Custaud, E. S. Tomilovskaya, I. M. Larina, T. Mano, G. Gauquelin-Koch, C. Gharib and I. B. Kozlovskaya, "Long-Term Dry Immersion: Review and Prospects," European Journal of Applied Physiology, Vol. 111, No. 7, 2011, pp. 1235-1260.

\section{doi:10.1007/s00421-010-1750-x}

[5] M. E. Widlansky, "The Danger of Sedenterism: Endothelium at Risk," Heart and Circulatory Physiology: American Journal of Physiology, Vol. 299, No. 2, 2010, pp. 243-244. doi:10.1152/ajpheart.00505.2010

[6] M. A. Iunusov, V. N. Orlov and T. V. Vinokhodova, "Effect of the Model of 'Dry' Immersion on the Indicators of Water-Electrolyte Metabolism and Aldosterone and Cortisol in the Plasma of Persons with Different Levels of Body Water," Kosmicheskaia Biologiia i Aviakosmicheskaia Meditsina, Vol. 19, No. 4, 1985, pp. 42-45.

[7] S. G. Ivanov and E. E. Bogomazov, "Dry' Immersion and Prospects of Its Use in Clinical Practice," Kosmicheskaia Biologiia i Aviakosmicheskaia Meditsina, Vol. 22, No. 5, 1988, pp. 4-6.

[8] S. G. Ivanov and L. I. Markova, "Use of a 'Dry' Immersion Method in the Treatment of Hypertensive Crisis," Kosmicheskaia Biologiia i Aviakosmicheskaia Meditsina, Vol. 24, No. 1, 1990, pp. 40-42.

[9] N. M. Navasiolava, V. de Germain, T. Levrard, I. M. Larina, I. B. Kozlovskaya, B. Diquet, A. Le Bouil, M. A. Custaud and J. O. Fortrat, "Skin Vascular Resistance in the Standing Position Increases Significantly after 7 Days of Dry Immersion," Autonomic Neuroscience, Vol. 160, No. 1-2, 2011, pp. 64-68. doi:10.1016/j.autneu.2010.10.003

[10] M. Coupé, M. Yuan, C. Demiot, Y. Q. Bai, S. Z. Jiang, Y. Z. Li, P. Arbeille, G. Gauquelin-Koch, T. Levrard, M. A. Custaud and Y. H. Li, "Low-Magnitude Whole Body Vibration with Resistive Exercise as a Countermeasure against Cardiovascular Deconditioning after 60 Days of Head-Down Bed Rest," American Journal of Physiology-Regulatory, Integrative and Comparative Physiology, Vol. 301, No. 6, 2011, pp. 1748-1754. doi:10.1152/ajpregu.00234.2011

[11] V. P. Krotov, T. E. Burkovskaia, M. A. Dotsenko, Iu. V. Gordeev, A. M. Nosovskiı̌ and N. A. Chel'naia, "Blood Plasma Volume Dynamics in Monkeys during Immersion," Aviakosmicheskaia i Ekologicheskaia Meditsina, Vol. 38, No. 3, 2004, pp. 30-36.

[12] N. M. Navasiolava, A. Pajot, Y. Gallois, L. Kh. Pastushkova, V. A. Kulchitsky, G. Gauquelin-Koch, I. B. Kozlovskaya, M. Heer, O. Hand, I. M. Larina and M. A. Custaud, "NT-ProBNP Levels, Water and Sodium Homeostasis in Healthy Men: Effects of 7 Days of Dry Immersion," European Journal of Applied Physiology, Vol. 111, No. 9, 2011, pp. 2229-2237. doi:10.1007/s00421-011-1858-7

[13] J. A. Krasney, "Head-Out Water Immersion: Animal Studies," In: M. J. Fregly and C. M. Blatteis, Eds., Handbook of Physiology, Environmental Physiology, Oxford University Press, Oxford, 1996, pp. 855-887.

[14] K. I. Gogolev, E. A. Aleksandrova and E. B. Shul'zhenko, "Comparative Assessment of Changes during Antiorthostatic Hypokinesia and Immersion in Man," Human Physiology, Vol. 6, No. 6, 1980, pp. 392-396.

[15] A. M. Chă̌ka and I. S. Balakhovskiı̌, "Changes in Plasma and Extracellular Fluid Volumes and Plasma Protein Mass under Conditions of Head-Down Tilt Hypokinesia 
and Immersion," Kosmicheskaia Biologiia $i$ Aviakosmicheskaia Meditsina, Vol. 16, No. 6, 1982, pp. 22-28.

[16] A. Bergouignan, I. Momken, D. A. Schoeller, S. Normand, A. Zahariev, B. Lescure, C. Simon and S. Blanc, "Regulation of Energy Balance during Long-Term Physical Inactivity Induced by Bed Rest with and without Exercise Training," Journal of Clinical Endocrinology \& Metabolism, Vol. 95, No. 3, 2010, pp. 1045-1053. doi: $10.1210 /$ jc. $2009-1005$

[17] S. Blanc, S. Normand, C. Pachiaudi, J. O. Fortrat, M. Laville and C. Gharib, "Fuel Homeostasis during Physical Inactivity Induced by Bed Rest," Journal of Clinical Endocrinology \& Metabolism, Vol. 85, No. 6, 2000, pp. 2223-2233. doi:10.1210/jc.85.6.2223

[18] N. M. Hamburg, C. J. McMackin, A. L. Huang, S. M. Shenouda, M. E. Widlansky, E. Schulz, N. Gokce, N. B. Ruderman, J. F. Keaney Jr. and J. A. Vita, "Physical Inactivity Rapidly Induces Insulin Resistance and Microvascular Dysfunction in Healthy Volunteers," Arteriosclerosis, Thrombosis, and Vascular Biology, Vol. 27, No. 12, 2007, pp. 2650-2656. doi:10.1161/ATVBAHA.107.153288

[19] M. Fenske, "Urinary Free Cortisol and Cortisone Excretion in Healthy Individuals: Influence of Water Loading," Steroids, Vol. 71, No. 11-12, 2006, pp. 1014-1018. doi:10.1016/j.steroids.2006.08.004

[20] J. C. Buckey Jr., L. D. Lane, B. D. Levine, D. E. Watenpaugh, S. J. Wright, W. E. Moore, F. A. Gaffney and C. G. Blomqvist, "Orthostatic Intolerance after Spaceflight," Journal of Applied Physiology, Vol. 81, No. 1, 1996, pp. 7-18.

[21] V. A. Convertino, "Exercise and Adaptation to Microgravity Environment," In: M. J. Fregly and C. M. Blatteis, Eds., Handbook of Physiology, Environmental Physiology, Oxford University Press, Oxford, 1996, pp. 815-843.

[22] V. A. Convertino, "Mechanisms of Microgravity Induced Orthostatic Intolerance: Implications for Effective Countermeasures," Journal of Gravitational Physiology, Vol. 9, No. 2, 2002, pp. 1-13.

[23] K. A. Engelke, D. F. Doerr, C. G. Crandall and V. A. Convertino, "Application of Acute Maximal Exercise to Protect Orthostatic Tolerance after Simulated Microgravity," American Journal of Physiology, Vol. 271, No. 4, 1996, pp. R837-R847.

[24] T. Mano, "Autonomic Neural Functions in Space," Current Pharmaceutical Biotechnology, Vol. 6, No. 4, 2005, pp. 319-324. doi:10.2174/1389201054553743
[25] A. I. Grigor'ev, I. B. Kozlovskaia and B. S. Shenkman, "The Role of Support Afferents in Organization of the Tonic Muscle System," Rossiǔskii Fiziologicheskiu Zhurnal Imeni I.M. Sechenova, Vol. 90, No. 5, 2004, pp. 508521.

[26] L. F. Zhang, "Vascular Adaptation to Microgravity: What Have We Learned?" Journal of Applied Physiology, Vol. 91, No. 6, 2001, pp. 2415-2430.

[27] M. Coupe, J. O. Fortrat, I. M. Larina, G. Gauquelin-Koch, C. Gharib and M. A. Custaud, "Cardiovascular Deconditioning: From Autonomic Nervous System to Microvascular Dysfunctions," Respiratory Physiology \& Neurobiology, Vol. 169, No. 1, 2009, pp. 10-12. doi:10.1016/j.resp.2009.04.009

[28] P. C. de Groot, M. W. Bleeker and M. T. Hopman, "Magnitude and Time Course of Arterial Vascular Adaptations to Inactivity in Humans," Exercise and Sport Sciences Reviews, Vol. 34, No. 2, 2006, pp. 65-71. doi:10.1249/00003677-200604000-00005

[29] V. A. Convertino, "Clinical Aspects of the Control of Plasma Volume at Microgravity and during Return to one Gravity," Medicine \& Science in Sports \& Exercise, Vol. 28, No. 10, 1996, pp. 45-52. doi:10.1097/00005768-199610000-00033

[30] E. F. Coyle, M. K. Hemmert and A. R. Coggan, "Effects of Detraining on Cardiovascular Responses to Exercise: Role of Blood Volume," Journal of Applied Physiology, Vol. 60, No. 1, 1986, pp. 95-99.

[31] W. W. Waters, S. H. Platts, B. M. Mitchell, P. A. Whitson and J. V. Meck, "Plasma Volume Restoration with Salt Tablets and Water after Bed Rest Prevents Orthostatic Hypotension and Changes in Supine Hemodynamic and Endocrine Variables," Heart and Circulatory Physiology: American Journal of Physiology, Vol. 288, No. 2, 2005, pp. 839-847. doi:10.1152/ajpheart.00220.2004

[32] J. Vernikos and V. A. Convertino, "Advantages and Disadvantages of Fludrocortisone or Saline Load in Preventing Post-Spaceflight Orthostatic Hypotension," Acta Astronaut, Vol. 33, 1994, pp. 259-266. doi:10.1016/0094-5765(94)90133-3

[33] M. A. Custaud, C. Millet, J. Frutoso, A. Maillet, G. Gauquelin, C. Gharib and J. O. Fortrat, "No Effect of Venoconstrictive Thigh Cuffs on Orthostatic Hypotension Induced by Head-Down Bed Rest," Acta Physiologica Scandinavica, Vol. 170, No. 2, 2000, pp. 77-85. doi:10.1046/j.1365-201x.2000.00763.x 


\section{Supplementary Data}

Table S1. Variations from baseline for creatinine, free water, osmolal and urea clearances $(\Delta=$ valueday - valuebaseline).

\begin{tabular}{cccccc}
\hline & DI 1 & DI 2 & DI 4 & R0 & R+2 \\
\hline$\Delta \mathrm{CCr}, \mathrm{ml} / \mathrm{min}$ & $5.6 \pm 7.0$ & $8.9 \pm 10.7$ & $-2.9 \pm 13.9$ & $-18.9 \pm 8.4$ & $4.9 \pm 5.8$ \\
$\Delta \mathrm{CH}_{2} \mathrm{O}, \mathrm{ml} / \mathrm{min}$ & $0.42 \pm 0.2$ & $0.61 \pm 0.3$ & $0.43 \pm 0.22$ & $0.45 \pm 0.2$ & $0.11 \pm 0.22$ \\
$\Delta \mathrm{Cosm}, \mathrm{ml} / \mathrm{min}$ & $-0.32 \pm 0.14$ & $-0.23 \pm 0.09$ & $-0.41 \pm 0.14^{*}$ & $0.08 \pm 0.19$ & $0.15 \pm 0.11$ \\
$\Delta \mathrm{Curea}, \mathrm{ml} / \mathrm{min}$ & $13.9 \pm 1.9^{*}$ & $6.8 \pm 3.8$ & $5.9 \pm 5.3$ & $-9.6 \pm 2.6^{*}$ & $5.4 \pm 3.3$ \\
\hline
\end{tabular}

$\mathrm{CCr}$ : creatinine clearance; $\mathrm{CH}_{2} \mathrm{O}$ : free water clearance; Cosm: osmolal clearance; Curea: urea clearance; B: baseline; DI: dry immersion; R: recovery; values are means $\pm \mathrm{SE}, \stackrel{*}{\mathrm{p}}<0.05$ vs baseline.

Table S2. Morning blood pressure and heart rate before, during and after dry immersion.

\begin{tabular}{|c|c|c|c|c|c|c|c|c|c|c|}
\hline & B-2 & B-1 & DI 1 & DI 2 & DI 3 & DI 4 & DI 5 & R0 & $\mathrm{R}+1$ & $\mathrm{R}+2$ \\
\hline SBP, mmHg & $124 \pm 3$ & $123 \pm 3$ & $121 \pm 3$ & $116 \pm 2$ & $121 \pm 2$ & $123 \pm 3$ & $113 \pm 2^{*}$ & $121 \pm 3$ & $123 \pm 2$ & $118 \pm 2$ \\
\hline DBP, mmHg & $72 \pm 2$ & $69 \pm 2$ & $71 \pm 2$ & $68 \pm 1$ & $73 \pm 2$ & $68 \pm 2$ & $70 \pm 2$ & $71 \pm 1$ & $68 \pm 2$ & $64 \pm 2$ \\
\hline $\mathrm{HR}, \mathrm{bpm}$ & $64 \pm 4$ & $65 \pm 4$ & $67 \pm 4$ & $57 \pm 2^{*}$ & $56 \pm 3^{*}$ & $61 \pm 3$ & $58 \pm 2$ & $57 \pm 2$ & $69 \pm 3$ & $71 \pm 2$ \\
\hline
\end{tabular}

B: baseline; DI: dry immersion; R: recovery; SBP: systolic blood pressure; DBP: diastolic blood pressure; HR: heart rate; values are means \pm SE. ${ }^{*} \mathrm{p}<0.05$ vs B-1. 